\title{
Stimulation of probiotic bacteria induces release of membrane vesicles with augmented anti-inflammatory activity
}

Lisann Müller, Thomas Kuhn, Marcus Koch and Gregor Fuhrmann*

\section{AUTHOR ADDRESS}

L. Müller, T. Kuhn, Dr. G. Fuhrmann

Helmholtz Institute for Pharmaceutical Research Saarland, Department of Pharmacy, Saarland University, Campus E8 1, 66123 Saarbrücken, Germany

Dr. M. Koch

INM - Leibniz Institute for New Materials, Campus D2 2, 66123 Saarbrücken

*gregor.fuhrmann@helmholtz-hips.de

\section{Supporting Figures}

Figure S1. Schematic overview of the isolation and characterization of probiotic membrane vesicles

Figure S2. Comparison of growth curves of L. casei, cultivated under agitation, anaerobic, pH 5 and $\mathrm{pH} 8$ condition

Figure S3. Growth curves of L. plantarum, cultivated under static, anaerobic, $\mathrm{pH} 5$ and $\mathrm{pH} 8$ condition

Figure S4. SEC column characterization for eluted fractions of L. casei and L. plantarum

Figure S5. Typical size distribution profile of isolated MVs produced by L. casei and L. plantarum under reference conditions

Figure S6. Measurement of the $\mathrm{pH}$-value of the bacterial cultures over the course of their growth

Figure S7. Additional cryo-transmission electron microscopy images 


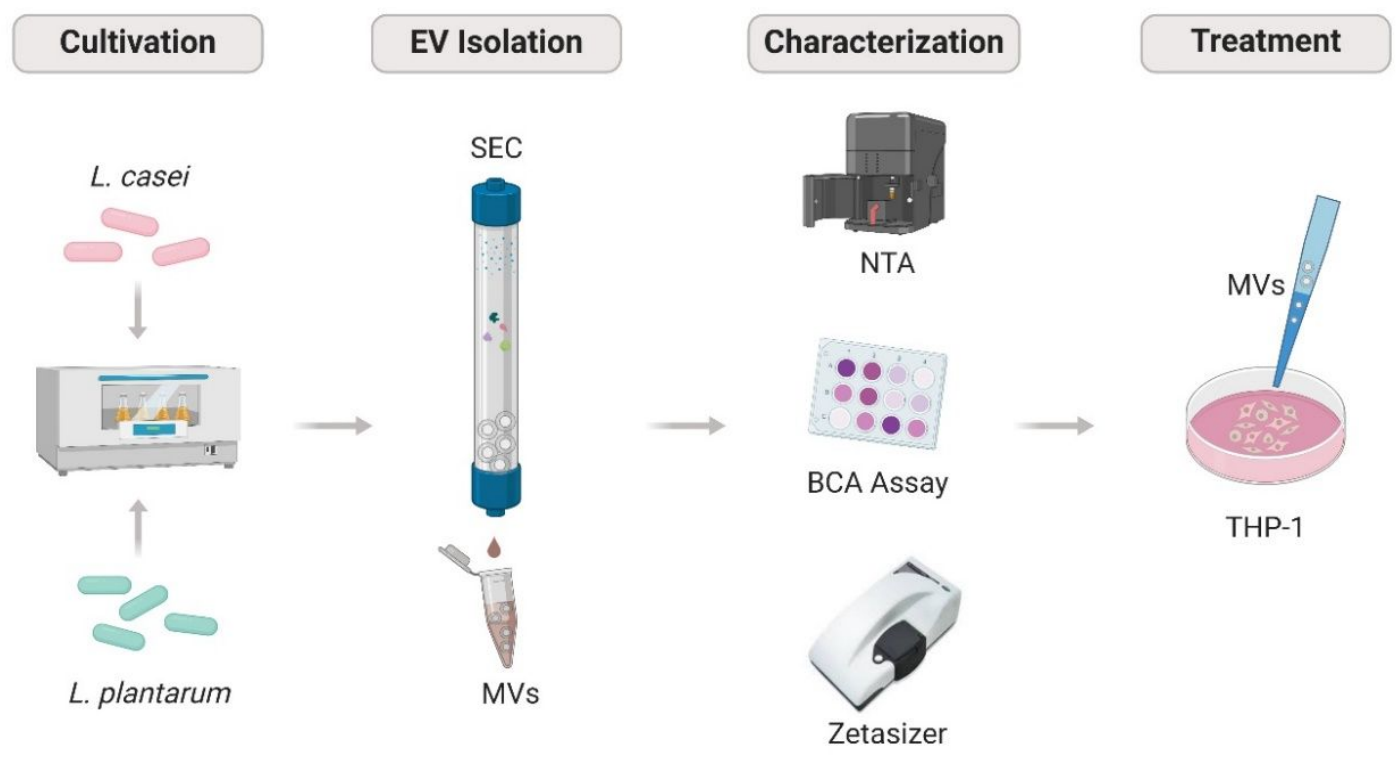

Figure S1. Schematic overview of the isolation and characterization of probiotic membrane vesicles. Different cultivation conditions of $L$. casei and $L$. plantarum were carried out to determine their influence on MV production. MVs derived under different conditions were isolated by means of SEC and characterized with different analysis methods, including nanoparticle tracking analysis, bicinchonic acid protein kit and Zetasizer analysis. The vesicles' anti-inflamatory activity was assessed on macrophage like cells treated by ELISA. [Own representation via BioRender]
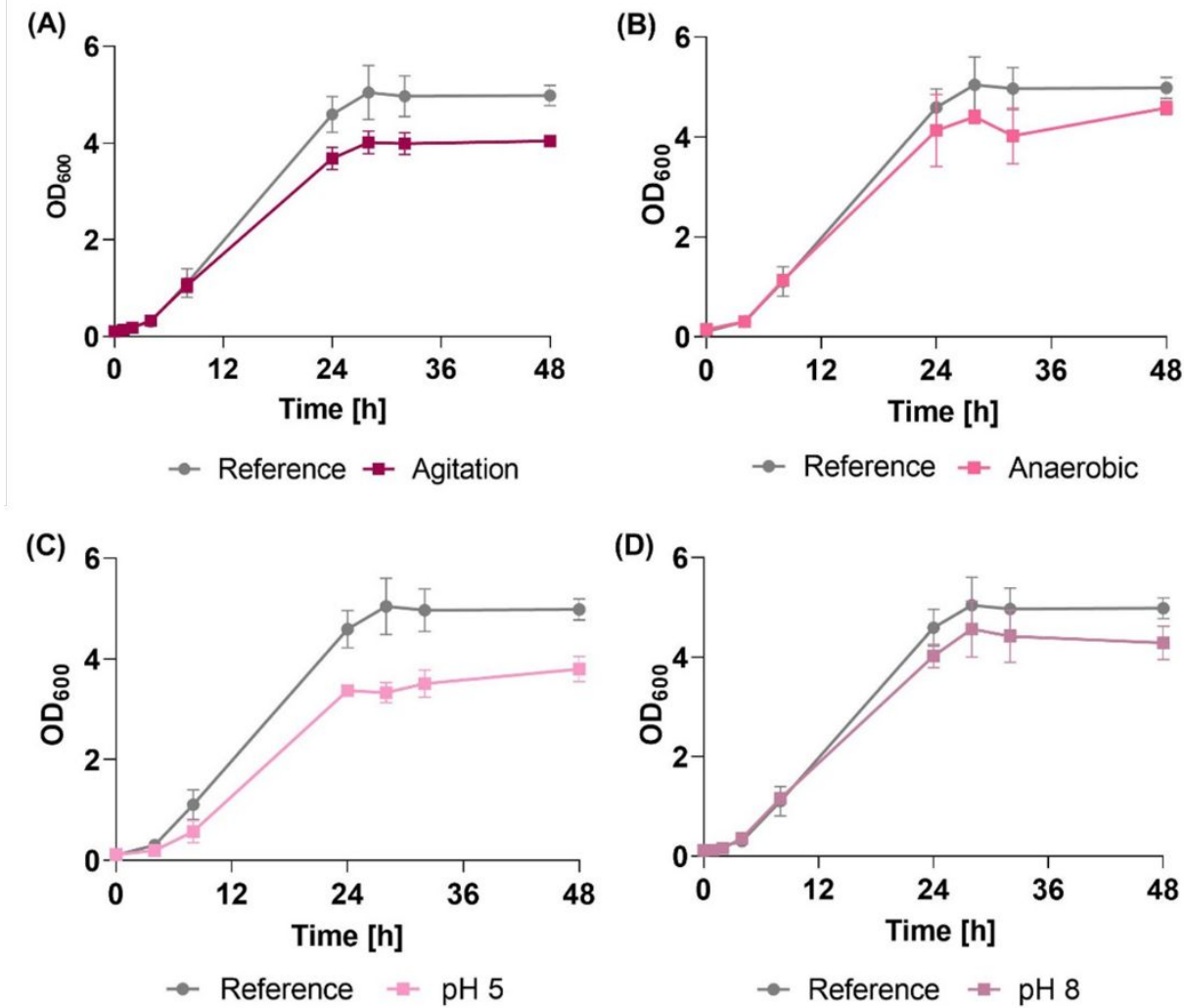

Figure S2. Comparison of growth curves of $L$. casei, cultivated under (A) agitation, (B) anaerobic, (C) pH 5 and (D) pH 8 condition. Grown at $37^{\circ} \mathrm{C}$ and $0 \mathrm{rpm}$ in MRS medium (reference), $180 \mathrm{rpm}$ (agitation), anaerobic flasks (anaerobic) or MRS medium adjusted to $\mathrm{pH} 5$ or 8 . OD measured at 600 $\mathrm{nm}$. Shown are mean values \pm SD from independent triplicates. 


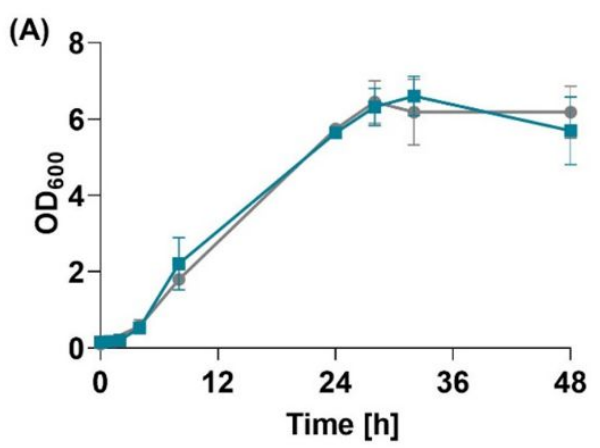

$\rightarrow$ Reference $\rightarrow$ Static

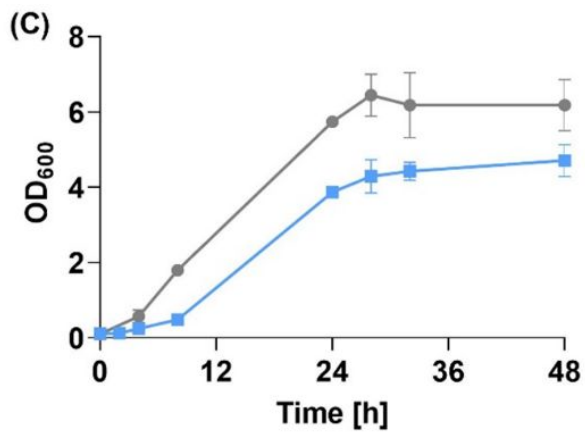

$\rightarrow$ Reference $\rightarrow$ pH 5

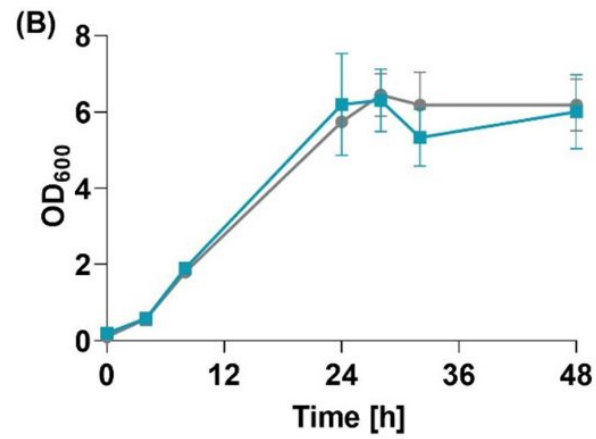

$\rightarrow$ Reference -- Anaerobic

(D)

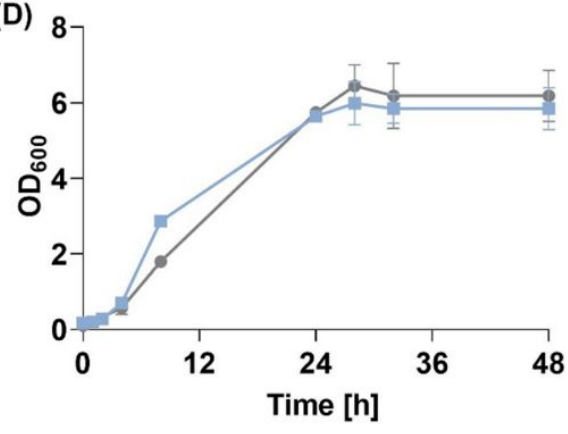

$\rightarrow$ Reference $\rightarrow-\mathrm{pH} 8$

Figure S3. Growth curves of $L$. plantarum, cultivated under (A) static, (B) anaerobic, (C) pH 5 and (D) pH 8 condition. Grown at $30^{\circ} \mathrm{C}$ and $180 \mathrm{rpm}$ in MRS medium (reference), $0 \mathrm{rpm}$ in MRS medium (static), anaerobic flasks (anaerobic) or MRS medium adjusted to $\mathrm{pH} 5$ or 8 . OD measured at $600 \mathrm{~nm}$. Shown are mean values \pm SD from independent triplicates.

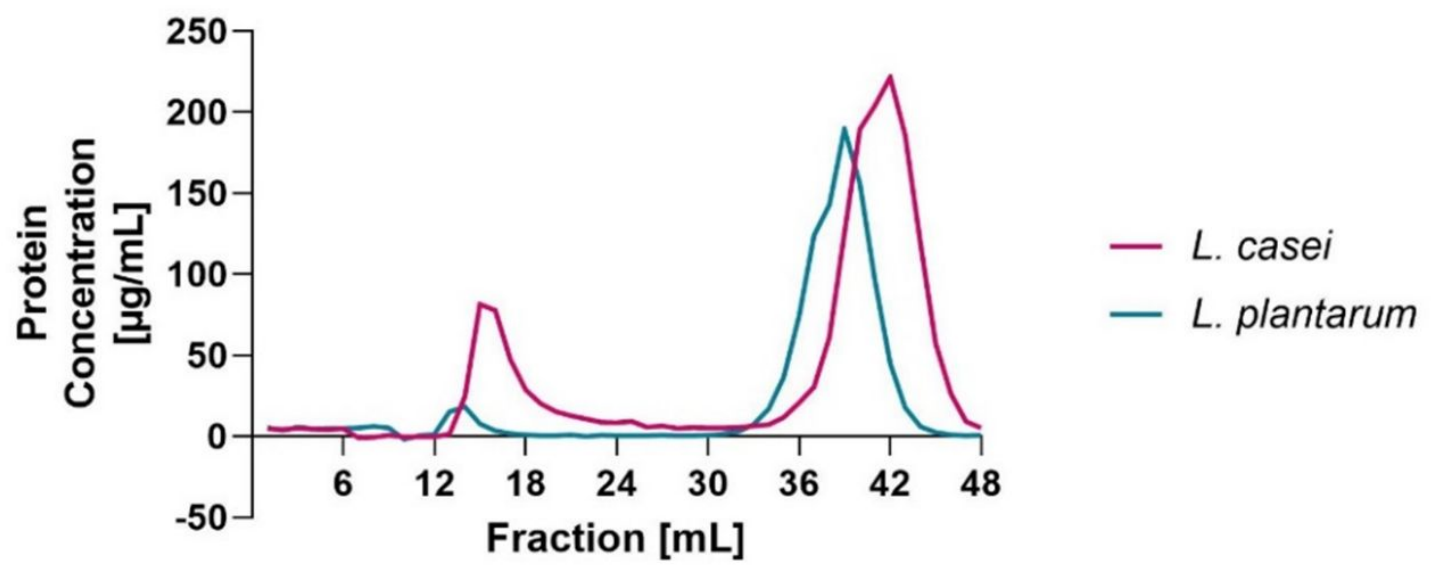

Figure S4. SEC column characterization for eluted fractions of $L$. casei and $L$. plantarum. Protein content was determined by BCA assay 

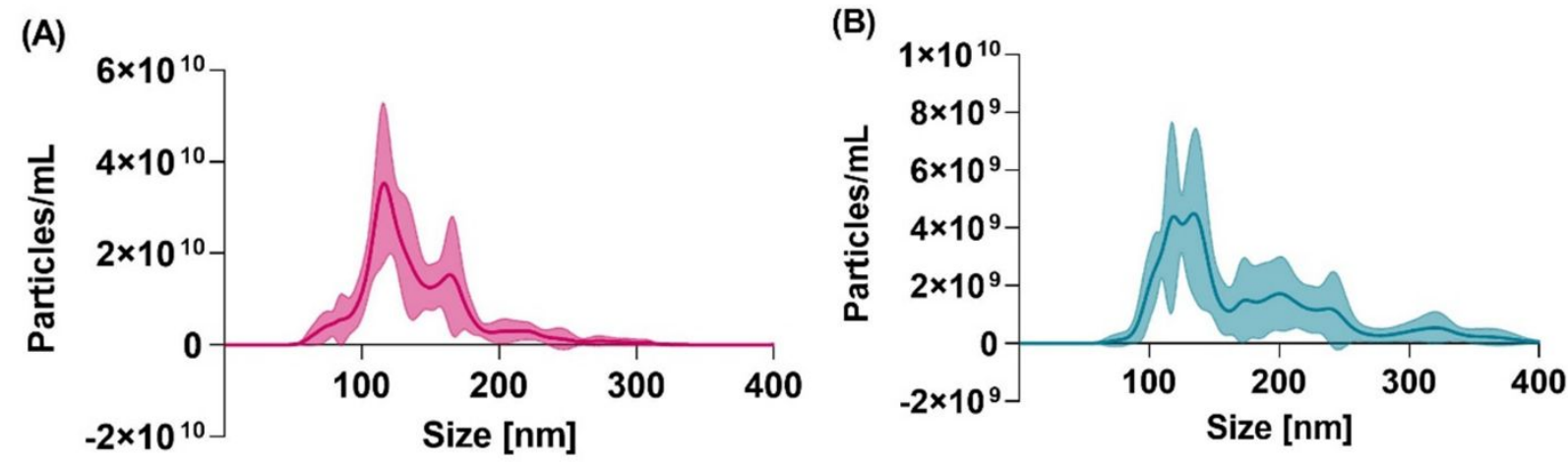

Figure S5. Typical size distribution profile of isolated MVs produced by (A) $L$. casei and (B) $L$. plantarum under reference conditions. Particle sizes and concentrations were obtained by NTA measurement. Shown are mean values \pm SD from $n \geq 3$ independent experiments; SD is demonstrated as colored area below and above mean values.
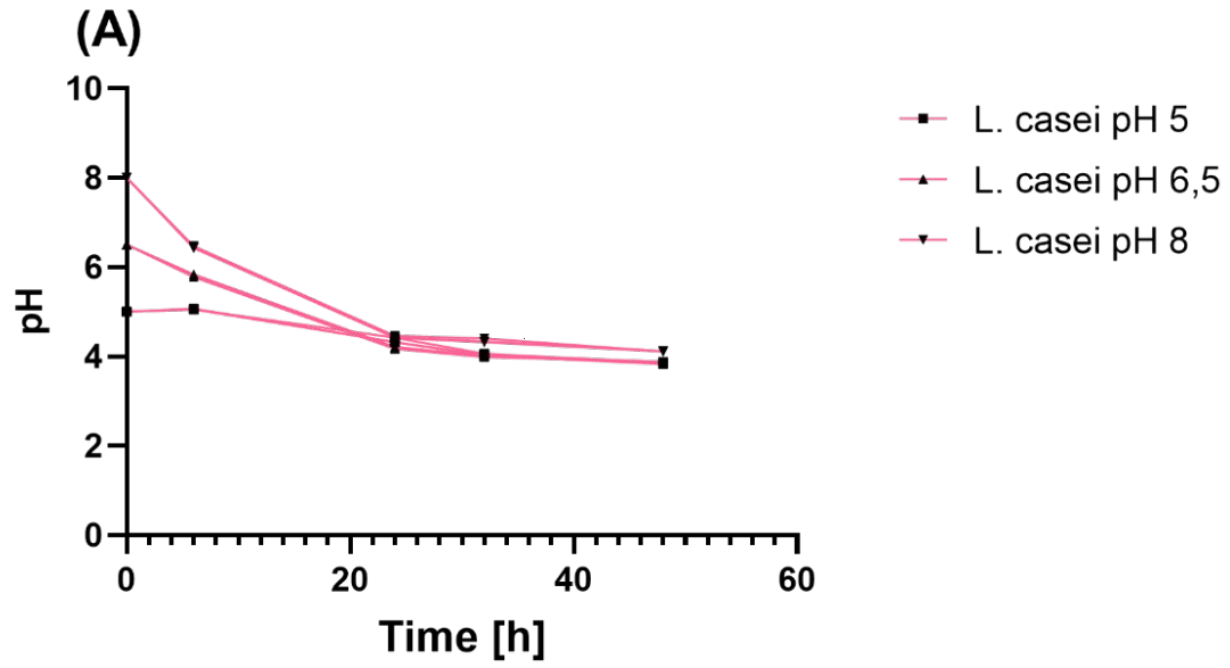

(B)

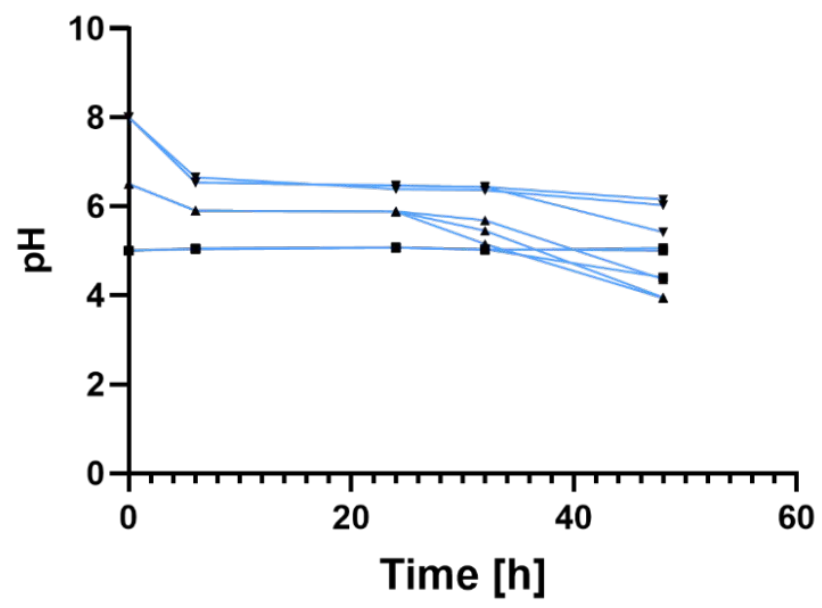

- L. plantarum pH 5

- L. plantarum $\mathrm{pH} 6,5$

$\rightarrow$ L. plantarum $\mathrm{pH} 8$

Figure S6. Measurement of the pH-value of the bacterial cultures over the course of their growth. For the different $\mathrm{pH}$-conditions, the $\mathrm{pH}$-change of the cultures of (A) L. casei and (B) L. plantarum was monitored. Individual values are represented by each data point. The experiment was performed in $\mathrm{n}=3$. 

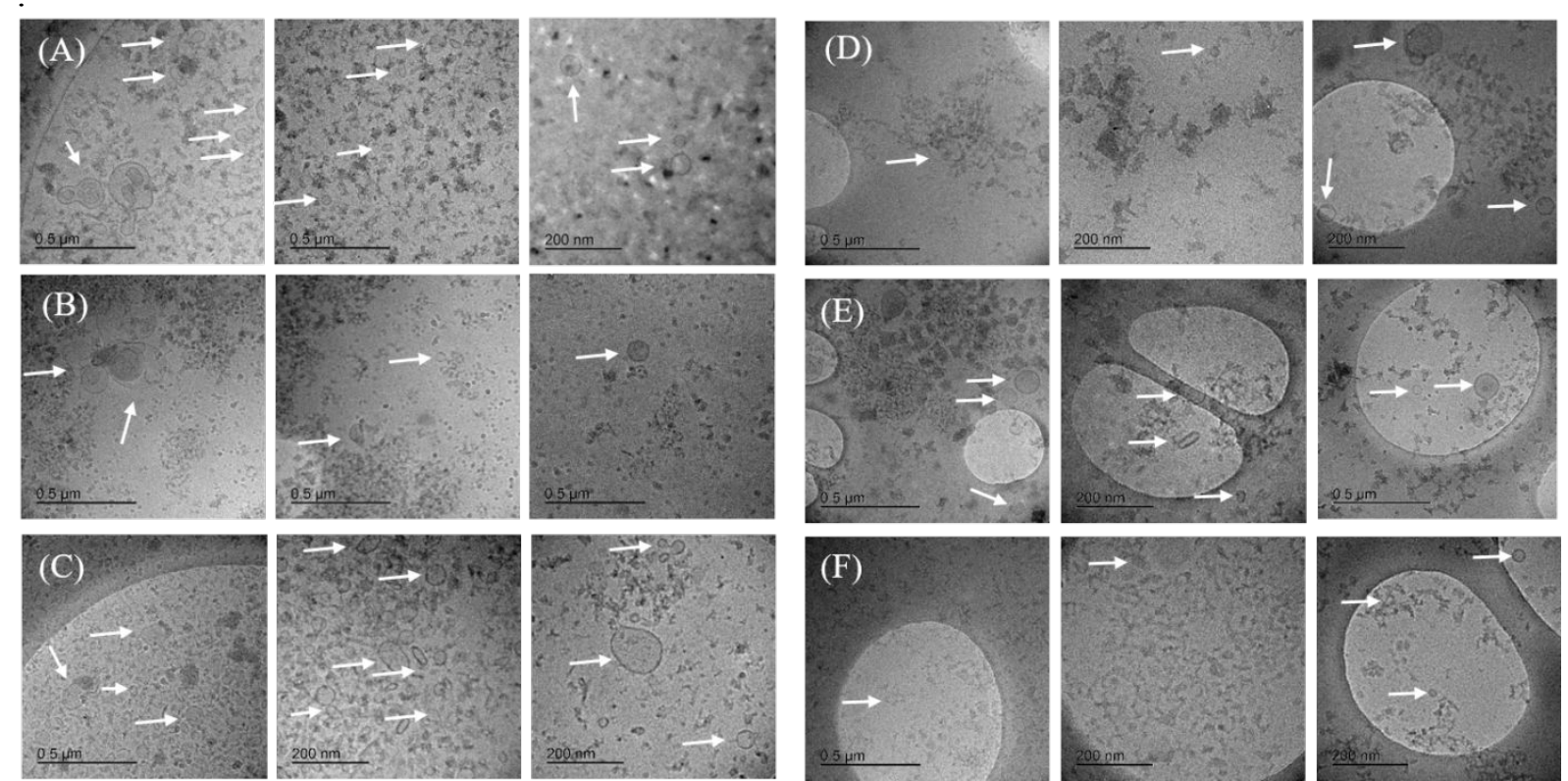

Figure S7. Additional cryo-transmission electron microscopy images of Lactobacillus MVs. (A) $L$. casei reference condition (B) L. plantarum reference condition (C) $L$. casei grown at $\mathrm{pH} 8$ (D) $L$. plantarum static growth condition (E) L. plantarum grown at $\mathrm{pH} 8$ (F) L. plantarum anaerobic growth condition.

(A)
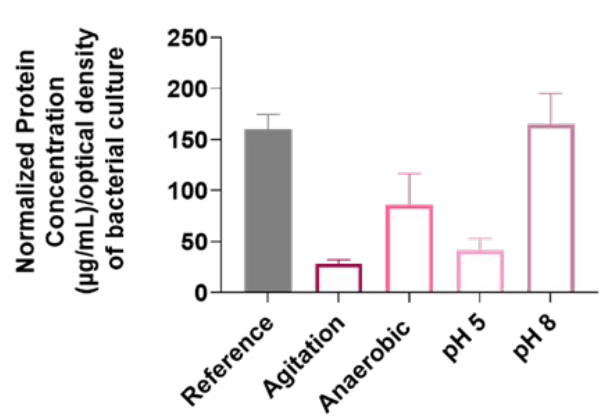

(C)
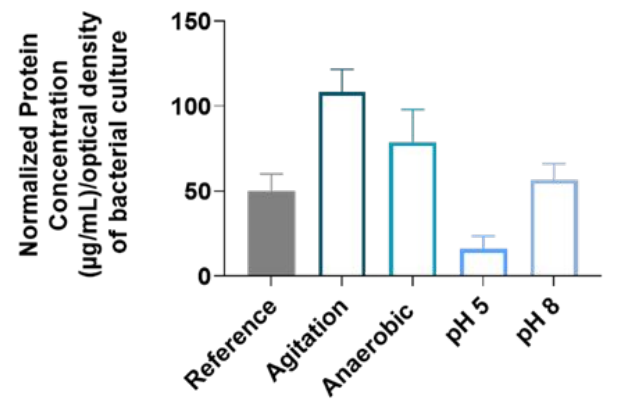

(B)

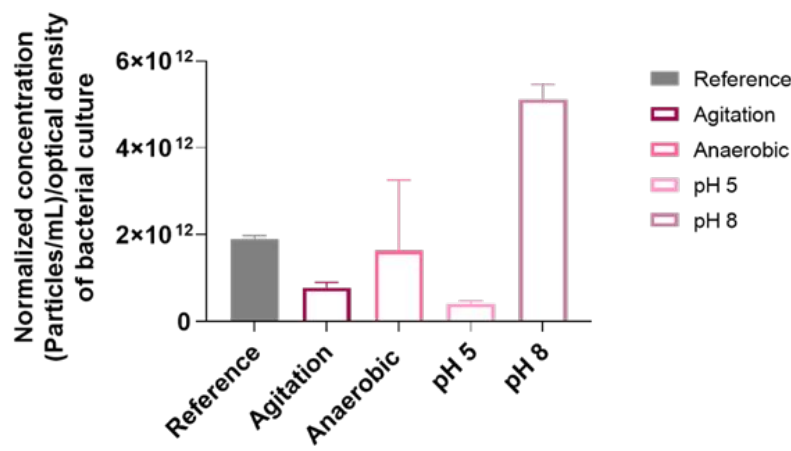

(D)

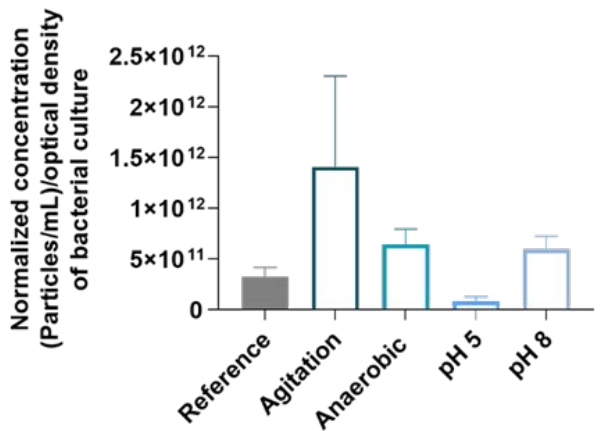

- Reference

ㅁ Agitation

ㅁ Anaerobic

$\square \mathrm{pH} 5$

$\square \mathrm{pH} 8$

Figure S8. Normalized protein and particle concentrations. Values were normalized to the optical density of the cultures. (A) and (B) protein and particle concentration of L. casei MVs, (C) and (D) protein and article concentration of L. plantarum MVs. Shown are mean values \pm SD from independent triplicates. 